\title{
Low-Cost Covid-19 Screening Protocol
}

\author{
Mohamed A. Ghanem \\ Department of Anesthesia and Surgical Intensive care, Faculty of Medicine, \\ Mansoura University, Egypt.
}

Corresponding Author Mohamed A. Ghanem

Mobile:

002-01067883998

E mail:

mohamed.abdel_latif@ yahoo.com.

Key words: Low-Cost; Covid-19; Screening ;Protocol
Background: COVID19 still not under human medical control, still killing people every were, virus lethality is unpredictable, time needed till having an effective drug or vaccine is still unclear, and humanity economic resources are being exhausted. Low-cost COVID-19 screening program is of great importance all over the world due to early general inventory of COVID-19 +ve patients before the onset of pulmonary symptoms in highly susceptible population (city, village, hospital clinics...etc). Hypothesis: Blood picture changes in COVID-19+ve patients as

\section{INTRODUCTION}

The incubation period of Covid 19 is 4 (2-7) up to 14 days [8]. Typical severe disease (based on analysis of multiple studies by Forest Arnold ):Dyspnea 6 days post exposure, hospital admission after 8 days and ICU admission/intubation after 10 days post exposure. Some patients are stable for several days after admission, but subsequently deteriorate rapidly[9].

The most common reported symptoms at onset of illness were fever $(98.6 \%)$, fatigue $(69.6 \%)$, dry cough $(59.4 \%)$, myalgia (34.8\%), and dyspnea (31.2\%). Less common symptoms were headache, dizziness, abdominal pain, loss of taste or smell, diarrhea, nausea, and vomiting. About $10 \%$ of patients initially lymphocytopenia , increased Ddimer $>1000 \mu \mathrm{g} / \mathrm{L}(>1 \mu \mathrm{g} / \mathrm{ml})$ and mild elevation of serum Ferritin [early (survivors $>400 \mu \mathrm{g} / \mathrm{L}$ ) and late (nonsurvivors $>1000 \mu \mathrm{g} / \mathrm{L}$ )] occur early prior to pulmonary symptoms, and portable chest $\mathrm{X}$ ray hence can be used as reliable indicator for early COVID-19 diagnosis and this may save the use of CT chest as well as swab-PCR.

Conclusion: We can screen out COVID 19 endemic zones and run the outpatient clinics in hospitals with the least coast during the period of lack of effective drug or vaccine against COVID 19 epidemic.

presented with diarrhea and nausea 1 to 2 days prior to development of fever and dyspnea. No fever doesn't mean no COVID-19. [3]. Silent hypoxemia even respiratory failure may occur without dyspnea (especially elderly). Pharyngitis or tonsil enlargement may be present[4] (Figure 1 ).

Complete blood count importance in COVID 19: WBC count tends to be normal. Lymphopenia is common, seen in $\sim 80 \%$ of patients [5]. Cutoff of $<1,500 / \mathrm{mcl}(<1,100 / \mathrm{mcl}$ in some studies) in adults may increase sensitivity for diagnosis of COVID19[11]. Mild thrombocytopenia is common (but platelets are rarely $<100000 / \mathrm{mcl}$ ). Lower platelet count is a poor prognostic sign[6]. 
Figure 1: COVID 19 Staging[2];

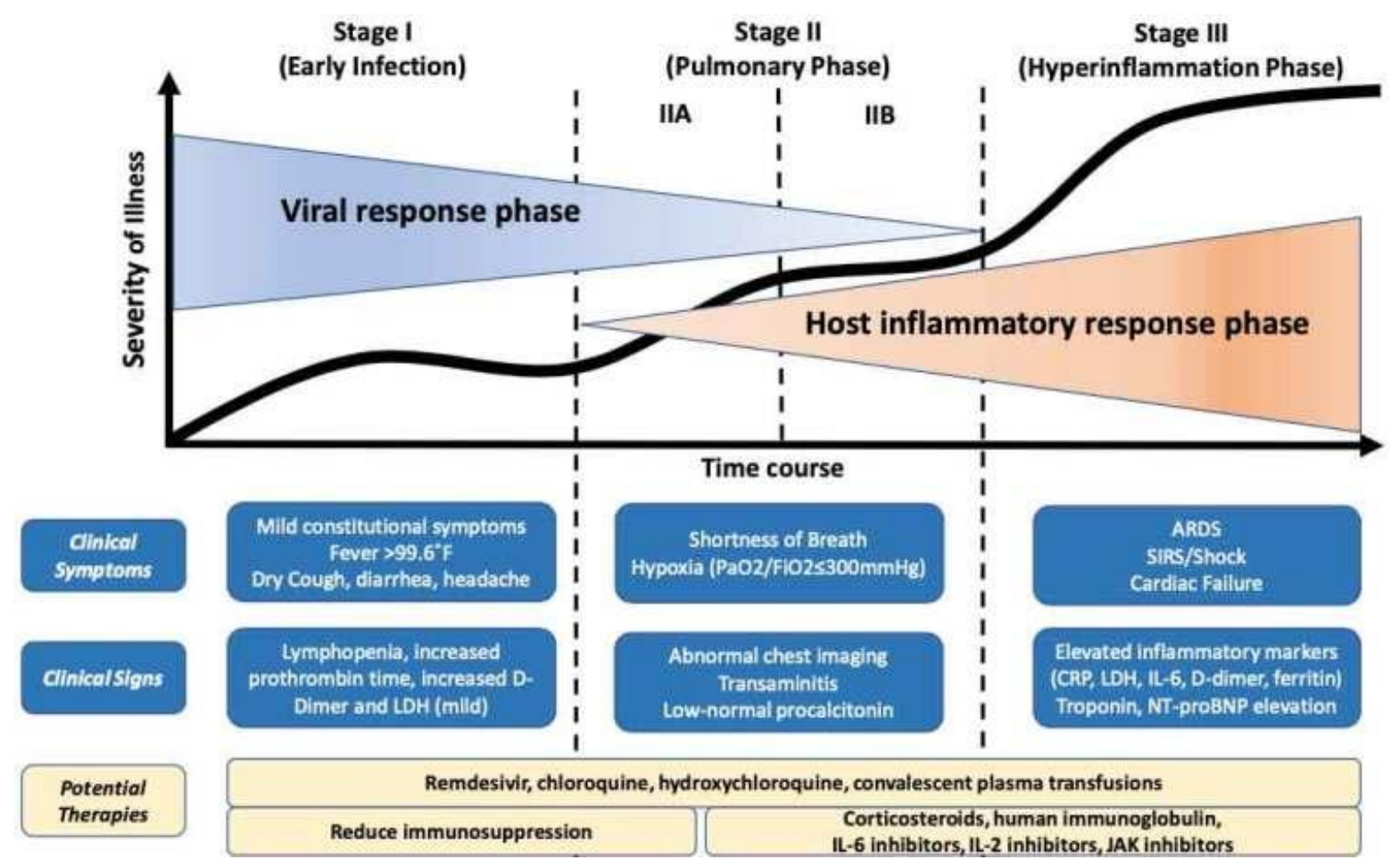

The above staging system was proposed by Siddiqi et al [2]. Patient courses may vary, making discrete staging challenging. However, this provides a useful conceptualization of the disease process.

Chest $\mathrm{X}$ ray: The sensitivity of chest X-ray was $59 \%$, compared to $86 \%$ for CT scan [4]. In a study done in Seattle Region, USA ; a chest radiograph was obtained in 23 patients $(96 \%)$ on ICU admission, and all the radiographs showed bilateral pulmonary opacities without pleural effusions. A computed tomographic (CT) scan of the chest was obtained in 5 patients $(21 \%)$; four of the scans showed bilateral ground glass opacities, and one showed pulmonary nodules[7]. Regarding atypical X-ray and CT imaging findings, bronchial wall thickening, thick strand-like and tortuous opacity, subpleural grid-like or honeycomb-like thickening of interlobular septum which is single or multiple, multiple patchy consolidations, less commonly mild pleural effusion or enlargement of mediastinal lymph nodes, could be seen. Single or multiple solid nodules or consolidated nodules in the center of lobule, surrounded by ground-glass opacities are another less common picture[10].Portable chest radiography may be considered to minimize the risk of crossinfection. In cases of high clinical suspicion for COVID-19, a positive CXR may obviate the need for CT. Additionally, CXR utilization for early disease detection may also play an important role in areas around the world with limited access to RT-PCR COVID testing[12]. Also chest X-ray is useful to prognosticate patients and avoid missing non-COVID pathology.

\section{Protocol Steps: (Figure 2: consort of application)}

\section{1- The implementing side of the idea:}

- For endemic foci: Health offices of the ministry of health in all the villages and cities of the republic. 
- As preliminary for patient admission in the hospitals, medical centers, and outpatient clinics.

\section{2- The crew implementing the idea:}

- For Endemic foci: (radiology technician, medical laboratory technician or nurse, and a car driver)

- Outpatient clinic in medical centers and hospitals (radiology technician, medical laboratory technician or nurse).

\section{Screening requirements:}

- Venous blood samples; for Lymphopenia [1], increased D-dimer $>1000 \mu \mathrm{g} / \mathrm{L}(>1 \mu \mathrm{g} / \mathrm{ml}) \quad$ [2] and high serum ferritin as a staging prognostic tool [early (survivors 500-700 $\mu \mathrm{g} / \mathrm{L}$ ) late (non-survivors $>1000 \mu \mathrm{g} / \mathrm{L}$ )], and portable chest $\mathrm{X}$ ray hence can be used as reliable indicator for early diagnosis (lower third ground glass appearance GGA) + a deep nasal or throat swab.

\section{4- The time of implementation of the idea:}

-For endemic foci: during the hours of curfew by the inspection cars previously specified in the endemic area.

-For outpatient clinic before the patient hospital admission.

\section{5- Targets of the protocol:}

It will never be available to target million people with PCR testing, to reduce the cost, in my protocol only one individual from each house will be investigated, who is the most out-ofhome individual and most in contact with others, often the head of the family; the father, or a working mother (if it is negative, the whole house is often negative - if it is positive we call the whole family for examination).

\section{6- Idea explanation:}

I) Venous blood samples for examination of Lymphopenia+ D-dimer + high Serum ferritin.
II) Chest X-ray (if possible)

III) A brief questionnaire on COVID-19 symptoms \& signs (dry cough, fever, sore throat, generalized weakness, body aches, abdominal pain, loss of taste or smell, diarrhea, nausea, vomiting, pulmonary symptoms such as breathing difficulty and shortness of breathing).

IV) Deep throat or nasal Swab.

(The swab to be withdrawn and kept until the blood picture, chest X-ray, and Covid-19 symptoms is examined by the doctors and if the blood test and symptoms are positive for the Covid-19 the swap will be activated; if it is positive all the house will be considered positive.If the blood test, X-ray and Covid-19 symptoms are negative, the swap will be discarded and the whole house is negative).

\section{7- Sample collection and analysis:}

I) For endemic areas: The collected samples throughout the curfew with the patient's name and address phone number are recorded on them, collected, and sent at the end of the day to the central laboratory responsible for conducting examinations and are examined by the competent doctors.

II) For hospital clinic: The collected samples with the patient's name and address phone number are recorded on them to be sent on time to a clinic lab and the results to be examined on time by doctors ,if query +ve for COVID19 the patient admission to the hospital will be cancelled and the patient will be referred by specific COVID19 ambulance to the COVID19 isolation hospital for further investigation according to the protocol of the ministry of health early before the appearance of pulmonary symptoms.

8-Covid 19 Positive cases home contacts will be referred to the COVID19 hospitals for further investigation and management according to the protocol of the ministry of health early before the appearance of pulmonary symptoms. 


\section{Figure 2: Protocol consort of application:}

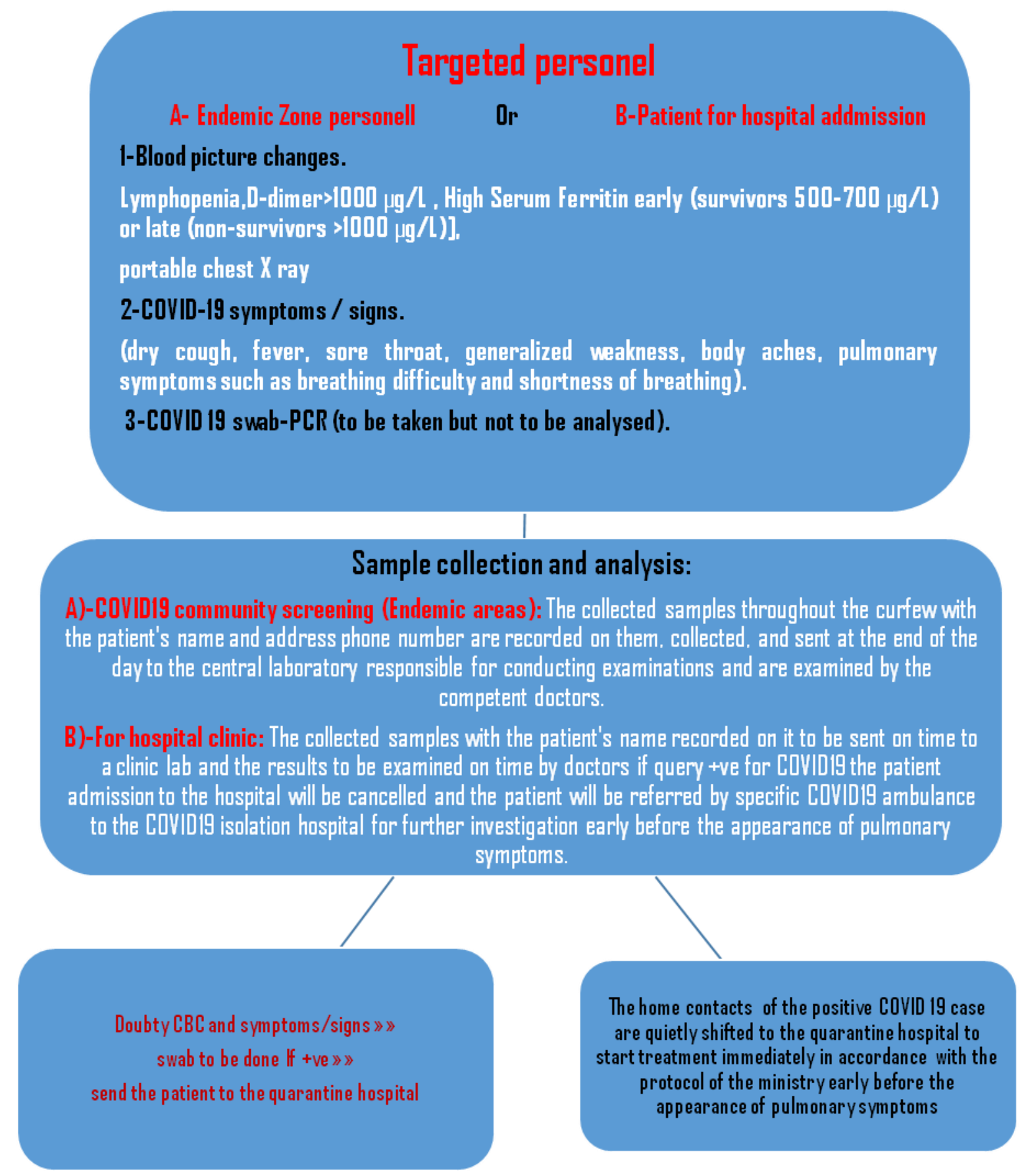

\section{REFERENCES}

1. Lippi G, Plebani M. Laboratory abnormalities in patients with COVID-2019 infection [published online ahead of print, 2020 Mar 3]. Clin Chem Lab Med. 2020;/j/cclm.ahead-of-print/cclm2020-0198/cclm-2020-0198.xml. doi:10.1515/cclm-2020-0198
The home contacts of the positive COVID 19 case are quietly shifted to the quarantine hospital to art treatment immediately in accordance with the protocol of the ministry early before the appearance of pulmonary symptoms
2. Siddiqi HK, Mehra MR. COVID-19 illness in native and immunosuppressed states: A clinicaltherapeutic staging proposal. J Heart Lung Transplant. 2020;39(5):405-407. doi:10.1016/j.healun.2020.03.012.

3. Dawei Wang D; Hu B; Hu C, MD; Zhu F; Xing Liu X; Zhang J, et al. Clinical Characteristics of 138 Hospitalized Patients With 2019 Novel

Ghanem, Afro-Egypt J Infect Endem Dis 2020;10(3):327-331

https://aeji.journals.ekb.eg/

http://mis.zu.edu.eg/ajied/home.aspx 
Coronavirus-Infected Pneumonia in Wuhan, China. JAMA 2020; 323(11):1061-1069. doi:10.1001/jama.2020.1585

4. Guan, Z. Ni, Yu Hu, W. Liang, C. Ou, J. He, L. Liu, et al. Clinical characteristics of coronavirus disease 2019 in China W. N Engl J Med 2020; 382:1708-20. DOI: 10.1056/NEJMoa2002032.

5. Yang X, Yu Y, Xu J,H, Xia J, Liu H, et al. Clinical course and outcomes of critically ill patients with SARS-CoV-2 pneumonia in Wuhan, China: a single-centered, retrospective, observational study [published correction appears in Lancet Respir Med. 2020 Apr;8(4):e26]. Lancet Respir Med. 2020;8(5):475-481. doi:10.1016/S22132600(20)30079-5

6. Ruan Q, Yang K, Wang W, Jiang L, Song J. Correction to: Clinical predictors of mortality due to COVID-19 based on an analysis of data of 150 patients from Wuhan, China. Intensive Care Med. 2020;46(6):1294-1297. doi:10.1007/s00134-020-06028-z

7. Bhatraju PK, Ghassemieh BJ, Nichols M, Kim R, Jerome KR, Nalla AK et al. Covid-19 in Critically Ill Patients in the Seattle Region - Case Series. N Engl J Med. 2020;382(21):2012-2022. doi:10.1056/NEJMoa2004500
8. del Rio C, Malani PN. COVID-19-New Insights on a Rapidly Changing Epidemic. JAMA. 2020;323(14):1339-1340. doi:10.1001/jama.2020.3072.

9. COVID-19 (SARS-COV-2) EPIDEMIC WITH DR. FOREST ARNOLD, February 28, 2020 / Victoria Roman. Louisville Lectures; Medical Lectures, University of Louisville School of Medicine.

10. Jin YH, Cai L, Cheng ZS, Cheng H, Deng T,Fan $\mathrm{YP}$, et al. A rapid advice guideline for the diagnosis and treatment of 2019 novel coronavirus (2019-nCoV) infected pneumonia(standard version). Mil Med Res 2020; 7(1):4.

11. Zhao Q, Meng M, Kumar R, Wu Y, Huang J, Deng $\mathrm{Y}$ et al. Lymphopenia is associated with severe coronavirus disease 2019 (COVID-19) infections: A systemic review and meta-analysis [published online ahead of print, 2020 May 4]. Int J Infect Dis. 2020;96:131-135. doi:10.1016/j.ijid.2020.04.086.

12. Jacobi A, Chung M, Bernheim A, Eber C. Portable chest X-ray in coronavirus disease-19 (COVID-19): A pictorial review. Clin Imaging. 2020;64:35-42. doi:10.1016/j.clinimag.2020.04.001 